\title{
MAXIMAL IMMEDIATE EXTENSIONS ARE NOT NECESSARILY MAXIMALLY COMPLETE
}

\author{
HANS HEINRICH BRUNGS and GÜNTER TÖRNER
}

(Received 4 May 1989)

Communicated by R. Lidl

\begin{abstract}
An extension $R_{1}$ of a right chain ring $R$ is called immediate if $R_{1}$ has the same residue division ring and the same lattice of principal right ideals as $R$. Properties of such immediate extensions are studied. It is proved that for every $R$, maximal immediate extensions exist, but that in contrast to the commutative case maximal right chain rings are not necessarily linearly compact.

1980 Mathematics subject classification (Amer. Math. Soc.) (1985 Revision): primary 16 A 20, 16 A 14 ; secondary 13 A 18.

Keywords and phrases: maximal valuation ring, completion, right chain ring.
\end{abstract}

\section{Introduction}

A commutative valuation ring $V$ that has no proper extension with the same group of values and the same residue field is called maximal (Krull [7]). Such valuation rings can also be characterized by the fact that every pseudo convergent sequence in $V$ has a limit in $V$ or that $V$ is linearly compact (Kaplansky [5]), that is, $V$ is maximally complete.

Here, we consider right chain rings $R$, that is, rings (not necessarily commutative) with identity for which $a R \subseteq b R$ or $b R \subset a R$ hold for any $a$, $b \in R$, and show that the above-mentioned notions carry over to this case.

After collecting some basic notations and results in Section 2 we define in Section 3 immediate extensions of right chain rings (see Definition 3.1)

The first author was supported in part by NSERC.

(C) 1990 Australian Mathematical Society 0263-6115/90\$A2.00+0.00 
discuss properties of such extensions and show that maximal immediate extensions exist (Theorem 3.6). In Section 4 we discuss pseudo convergent sequences and linear compactness, and show in particular that every element $z \in R_{1} \backslash R$ with $R_{1}$ an immediate extension of $R$, is a limit of a pseudo convergent sequence in $R$ (Theorem 4.12). Finally, in Section 5, we use some of the earlier results to show by an example that there exist right chain rings $R$ without proper immediate extensions, that is, maximal rings $R$, which are not maximally complete, that is, which contain pseudo convergent sequences that do not have a limit in $R$.

\section{Preliminaries}

All rings are assumed to be associative, with identity element, but not necessarily commutative. The Jacobson radical of a ring $R$ is denoted by $J(R)=J$ and the group of units of $R$ by $U(R)=U$. A ring is said to be a right chain ring if its lattice of right ideals is linearly ordered by inclusion, that is, for each $a, b \in R$ we have $a R \subseteq b R$ or $b R \subset a R$. Left chain rings are defined similarly and a left right chain ring is called a chain ring.

A domain is a ring without zero-divisors. Chain domains are the valuation rings studied in Mathiak [8]. A ring is ring invariant if all its right ideals are two-sided and invariant if it is right and left invariant. The valuation rings in Schilling [10] are exactly the invariant chain domains; these are the rings corresponding to valuations for skew fields into ordered groups.

A two-sided ideal $P$ of a ring $R$ is called prime if $a R b \subseteq P$ implies $a \in P$ or $b \in P$ in $P$, for $a, b \in R$, and completely prime (c.prime) if this implication follows from $a b \in P$.

In case $Q$ is a prime but not c.prime ideal of a right chain ring $R$, a c.prime ideal $P$ exists in $R$ with $P \supset Q$ such that there is no prime ideal between $P$ and $Q$ and $P^{2}=P$ (Bessenrodt et al. [1]). The question whether such prime ideals $Q$ can exist in chain rings is interesting and Dubrovin [4] constructed some examples; however it is difficult to follow his arguments at some places.

\section{Immediate extensions}

Krull defined immediate extensions of commutative valuation domains in [7] and showed that maximal immediate extensions exist. For right chain rings the following definition generalizes Krull's original definition. 
Definition 3.1. Let $R$ be a right chain ring. A ring extension $R_{1} \supseteq R$ is called an immediate extension of $R$ if the following conditions hold.

(i) For every element $a_{1} \in R_{1}$ there exist $a \in R, u_{1} \in U\left(R_{1}\right)$ with $a_{1}=a u_{1}$.

(ii) $\phi(r+J(R))=r+J\left(R_{1}\right)$ defines an isomorphism from the skew field $R / J(R)$ onto $R_{1} / J\left(R_{1}\right)$.

Before we list properties of immediate extensions we make a general observation about extensions of right chain rings and characterize immediate extensions by somewhat different conditions.

LEMMA 3.2. The following conditions are equivalent for right chain rings $R \subseteq R_{1}$ :

(a) $U\left(R_{1}\right) \cap R=U(R)$;

(b) $J\left(R_{1}\right) \cap R=J(R)$.

Proof. We observe that $U(R)=R \backslash J(R), U(R) \subseteq U\left(R_{1}\right) \cap R$ and $J\left(R_{1}\right) \cap R \subseteq J(R)$. Assume (a) and it follows that the element $j \in J(R)$ is also in $J\left(R_{1}\right)$, since $j$ would otherwise be in $U\left(R_{1}\right) \cap R=U(R)$. Similarly, under the assumption of (b), an element $v \in U\left(R_{1}\right) \cap R$ cannot be in $J(R)$, since it would then be in $J\left(R_{1}\right)$.

LEMMA 3.3. Let $R$ be a right chain ring. An extension $R_{1} \supseteq R$ is an immediate extension if and only if $U\left(R_{1}\right) \cap R=U(R)$ and for every element $a_{1} \in R_{1}$ there exist $a \in R$ and $j_{1} \in J\left(R_{1}\right)$ with $a_{1}=a\left(1+j_{1}\right)$.

Proof. We assume that $R_{1}$ satisfies the conditions of the lemma and (ii) of Definition 3.1 follows immediately, since $1+j_{1} \in U\left(R_{1}\right)$. To prove (b) define $\phi\left(a_{1}\right)=a+J(R) \in R / J(R)$ if $a_{1}=a\left(1+j_{1}\right) \in R_{1}$, with $a \in R$, $j_{1} \in J\left(R_{1}\right)$. This mapping defines a homomorphism from $R_{1}$ onto $R / J(R)$ with kernel $J\left(R_{1}\right)$ and it follows that the mapping $\phi$ in (b) of Definition 3.1 exists.

To prove the converse we observe first that an immediate extension of a right chain ring is again a right chain ring. It follows from (b) in Definition 3.1 that $J(R)=R \cap J\left(R_{1}\right)$ and hence $U(R)=U\left(R_{1}\right) \cap R$ by the above lemma. Finally, if $a_{1}=a^{\prime} u_{1}$ for $a_{1} \in R_{1}, a^{\prime} \in R$ and $u_{1} \in U\left(R_{1}\right)$ then $u_{1}=u+j_{1}^{\prime}$ for $u \in U(R), j_{1}^{\prime} \in J\left(R_{1}\right)$ and $a_{1}=a^{\prime} u\left(1+u^{-1} j_{1}^{\prime}\right)$ shows that the conditions of the lemma are satisfied.

Proposition 3.4. Let $R_{1}$ be an immediate extension of a right chain ring $R$. Then the following are satisfied.

(i) $R_{1}$ is a right chain ring. 
(ii) $J\left(R_{1}\right) \cap R=J(R)$.

(iii) $U\left(R_{1}\right) \cap R=U(R)$.

(iv) $\chi(a R)=a R_{1}$ defines a one-to-one mapping from the lattice $\mathscr{W}(R)$ of principal right ideals onto $\mathscr{W}\left(R_{1}\right)$.

(v) $R_{2}$ is an immediate extension of $R_{1}$ if and only if $R_{2}$ is an immediate extension of $R$ containing $R_{1}$.

(vi) Let $I$ be a right ideal in $R$. Then $I_{1}=\left\{\right.$ ir $\left.\mid i \in I, r \in R_{1}\right\}=I R_{1}$ is a right ideal in $R_{1}$ and $I R_{1} \cap R=I$.

(vii) Let $I_{1}$ be a right ideal in $R_{1}$. Then the contracted right ideal $I=$ $I_{1} \cap R$ satisfies $I_{1}=I R_{1}$.

(viii) The contraction of a two-sided ideal in $R_{1}$ is two-sided in $R$; the contraction of a c.prime ideal in $R_{1}$ is c.prime in $R$.

(ix) The contraction $I=I_{1} \cap R$ is idempotent in $R$ if $I_{1}$ is an idempotent ideal in $R_{1}$.

Proof. The statements (i)-(iii) follow from Lemma 3.3. That the mapping $\chi$ is onto follows from (i) in Definition 3.1 and if $a=b c$ in $R$ and $a R_{1}=b R_{1}$, then $a=b c=b u_{1}$ for $u_{1} \in U\left(R_{1}\right)$. Hence, $b\left(c-u_{1}\right)=0$ implies $b=0$ if $c \in J(R) \subseteq J\left(R_{1}\right)$.

(v) For an element $a_{2} \in R_{2}$ we have $a_{2}=a_{1} u_{2}=a u_{1} u_{2}$ for $a_{1} \in R_{1}$, $u_{2} \in U\left(R_{2}\right), u_{1} \in U\left(R_{1}\right), a \in R$. The skew fields of residues of $R$ and $R_{2}$ are also identical.

To prove (vi) let $\sum_{k=1}^{n} i_{k} r_{k}$ be an element in $I R_{1}$ with $i_{j} \in I, r_{j} \in R_{1}$, and we can assume $i_{j}=i_{1} a_{j}$ for $a_{j} \in R$ and all $j$. We have $\sum i_{k} r_{k}=$ $i_{1} \sum a_{k} r_{k}=i_{1} r$ for some $r \in R_{1}$ which shows that $I_{1}=I R_{1}$ is indeed a right ideal in $R_{1}$. An element in $I R_{1} \cap R$ has the form $i r u_{1}$ for $i \in I$, $r \in R, u_{1} \in U\left(R_{1}\right)$. If such an element is not contained in $I$, there exists $q \in J(R)$ with $\operatorname{iru}_{1} q=i$ and $i=0$ follows, a contradiction.

(vii) The inclusion $I R_{1} \subseteq I_{1}$ is obvious. Conversely, let $i u \in I_{1}$ with $i \in R, u \in U\left(R_{1}\right)$ and $i \in I, i u \in I R_{1}$ follows.

(viii) is obvious.

(ix) We can assume that $I_{1}$ and $I$ are nonzero. Nonzero idempotent ideals are c.prime and it follows that $I_{1}$ and hence $I$ are c.prime. If $I^{2} \subset I$ there exists an element $p \in I \backslash I^{2}$ and $p=p_{1} u_{1} p_{2} u_{2} \in I_{2}=I_{1}^{2}$ for $p_{1}$, $p_{2} \in I, u_{1}, u_{2} \in U\left(R_{1}\right)$. We have $u_{1} p_{2}=p_{2}^{\prime} u_{1}^{\prime}$ with $u_{1}^{\prime} \in U\left(R_{1}\right), p_{2}^{\prime} \in R$ and $p_{2}^{\prime} \in I$ follows. Hence, $p R_{1}=p_{1} p_{2}^{\prime} R_{1}$ and $p R=p_{1} p_{2}^{\prime} R$ by (iv). This implies $p \in I^{2}$, a contradiction.

The next result is the crucial step in the proof that maximal immediate extensions exist for right chain rings. The original proof of this result (see 
Krull [7]) For commutative valuation domains cannot be as easily adapted to our situation as the arguments in Rayner [9].

LEMMA 3.5. Let $R$ be a right chain ring, $R / J(R)=D, \mathscr{W}=\{a R \mid 0 \neq$ $a \in R\}$ and $R_{1}$ an immediate extension of $R$. Then $\left|R_{1}\right| \leq\left. D\right|^{\mathscr{W}}$.

Proof. Let $\mathscr{C}=\left\{c_{i} \mid c_{i} \in R, i \in T\right\}$ be a set of representatives of $D$ in $R$ and let $W^{\prime}$ be a subset of $R$ containing exactly one generator for each nonzero principal right ideal of $R$, that is $\mathscr{W}=\left\{a R \mid a \in W^{\prime}\right\}$ and $a R \neq a^{\prime} R$ for $a \neq a^{\prime}$ in $W^{\prime}$. Let $a \in W^{\prime}$ and $a R_{1}$ be the corresponding principal right ideal in $R_{1}$. We have $R_{1}=\bigcup_{i \in T}\left(c_{i}+J\left(R_{1}\right)\right)$, and $a R_{1}-\bigcup_{i \in T}\left(a c_{i}+a J\left(R_{1}\right)\right)$. Observe that $a\left(c_{i}-c_{j}\right) \neq a J\left(R_{1}\right)$ since $c_{i}-c_{j} \in U(R)$ for $i \neq j$. For $a R_{1}$, considered as a subgroup of the additive group $\left(R_{1},+\right)$, choose coset representatives $S=\left\{a_{j} \mid j \in L\right\}$, and $R_{1}=\bigcup_{j \in L}\left(a_{j}+a R_{1}\right)=$ $\bigcup_{i \in T, j \in L}\left(a_{j}+a c_{i}+a J\left(R_{1}\right)\right)$ follows. Let $r$ be any element in $R_{1}$ and consider in $\left(R_{1},+\right)$ the coset of $a J\left(R_{1}\right)$ that contains $r$, that is, $r+a J\left(R_{1}\right)=$ $a_{j}+a c_{i}+a J\left(R_{1}\right)$ for a certain $j \in L, i \in T$. We define a mapping $f$ from $R_{1}$ into $\mathscr{C}^{\mathscr{W}}$ by defining $f(r)=\bar{r} \in \mathscr{C}^{\mathscr{W}}$ with $\bar{r}(w)=c_{i}$ if $w=a R$ in $\mathscr{W}$. By construction, $f$ is well defined and we show that $f$ is one-to-one.

Let $r \neq t$ be elements in $R_{1}$ and $(r-t) R_{1}=a R_{1}$ follows a certain $a \in W^{\prime}$. We have $r+a R_{1}=t+a R_{1}$, but $r+a J\left(R_{1}\right) \neq t+a J\left(R_{1}\right)$, since $r-t=a u, u \in U\left(R_{1}\right)$. Hence, $r+a J\left(R_{1}\right)=a_{j}+a c_{1}+a J\left(R_{1}\right)$ and $t+a J\left(R_{1}\right)=a_{j}+a c_{2}+a J\left(R_{1}\right)$ where $a_{j} \in S$ and $c_{1} \neq c_{2} \in \mathscr{C}$. This implies $\bar{r}(w)=c_{1} \neq c_{2}=\bar{t}(w)$ for $w=a R$ and proves the lemma.

As a corollary we obtain

THEOREM 3.6. Any right chain ring $R$ has maximal immediate extensions.

Proof. We consider (using Lemma 3.5) the set $\mathscr{E}$ of immediate extensions. Let $R \subseteq R_{1} \subseteq R_{2} \subseteq \cdots$ be an ascending chain of immediate extensions of $R$ and $\widehat{R}=\bigcup_{i} R_{i}$ their union. We must show that $\widehat{R}$ is in $\mathscr{E}$.

If $a, b \in \widehat{R}$, then $a, b \in R_{i}$ some $i$ and $a c=b$ or $a=b c$ for some $c$ in $R_{i}$. Hence, $\widehat{R}$ is a right chain ring. We have $J(\widehat{R}) \cap R \subseteq J(R)$ and if $j \in J(R)$ is not in $J(\widehat{R})$, it is a unit in some $R_{i}$, a contradiction, since $J\left(R_{i}\right) \cap R=J(R)$ (Lemmas 3.2, 3.3). Finally, every $\hat{a} \in \widehat{R}$ is in some $R_{i}$ and $\hat{a}=a\left(1+j_{i}\right), j_{i} \in J\left(R_{i}\right)$. But $J(\widehat{R})=\bigcup_{i} J\left(R_{i}\right)$, since $U(\hat{R})=\bigcup_{i} U\left(R_{i}\right)$ and $\widehat{R} \in \mathscr{E}$ follows from Lemma 3.3.

We consider properties of a right chain ring $R$ that are inherited by immediate extensions. 
Proposition 3.7. Let $R_{1}$ be an immediate extension of the right chain ring $R$. Then the following conditions are satisfied.

(i) $I R_{1}$ is a prime ideal in $R_{1}$ if $I$ is a prime ideal in $R$ and $I R_{1}$ is a two-sided ideal in $R_{1}$.

(ii) $R_{1}$ is prime if $R$ is prime.

(iii) Let $I$ be a two-sided ideal in $R$ with $I_{1}=I R_{1}$ a two-sided ideal in $R_{1}$. Then $I$ is c.prime if and only if $I_{1}$ is c.prime.

(iv) $R$ is a domain if and only if $R_{1}$ is a domain.

(v) $R$ is right noetherian if and only if $R_{1}$ is right noetherian.

Proof. To prove (i) let $x_{1}=x u_{1}, y_{1}=y v_{1}$ be elements in $R$ with $x, y \in R, u_{1}, v_{1} \in U\left(R_{1}\right)$ and $x u_{1} R_{1} y v_{1} \subseteq I R_{1}$. Then $I R_{1} \supseteq x u_{1} R_{1} y=$ $x R_{1} y \supseteq x R y$ and $x R y \subseteq I R_{1} \cap R=I$ follows (Proposition 3.4(vii)). We have $x \in I$ or $y \in I$ which shows $x_{1} \in I_{1}$ or $y_{1} \in I_{1}$.

(ii) is obvious using (i) and $I=(0)$.

(iii) Suppose $I$ is completely prime and $x u_{1} y v_{1} \in I R_{1}$ with $x_{1}=x u_{1}$, $y_{1}=y v_{1} \in R_{1}, x, y \in R, u_{1}, v_{1} \in U\left(R_{1}\right)$. If $y v_{1} \in I R_{1}$ we are dome, otherwise $u_{1} y=y^{\prime} u_{1}^{\prime} \neq I R_{1}$ for $y^{\prime} \in R, u_{1}^{\prime} \in U\left(R_{1}\right)$. Hence, $y^{\prime} \in R \backslash I$, but $x u_{1} y v_{1}=x y^{\prime} u_{1}^{\prime} v_{1} \in I R_{1}$ implies $x y^{\prime} \in I$ and $x \in I$ follows. The converse is obvious and (iv) follows immediately.

To prove ( $v$ ) we observe that a right chain ring is right noetherian if and only if the maximum condition holds for principal right ideals. By Proposition 3.4(iv) this condition holds for $R$ if and only if it holds for $R_{1}$.

Right noetherian right chain rings are right invariant. We will show by an example that this is no longer true for discrete right chain rings where we use the following definition.

Definition 3.8. A right chain ring $R$ is called discrete if $R$ satisfies a.c.c. for c.prime ideals and $P \neq P^{2}$ for every proper c.prime ideal $P$.

LEMMA 3.9. Let $R$ be a discrete right chain ring. Then every prime ideal of $R$ is completely prime and $R_{P} / Q R_{P}$ is a right noetherian right chain ring with $Q$ the maximal prime ideal properly contained in $P$. The ring $R_{P}$ is a right noetherian right chain ring provided $P$ is the minimal prime ideal of $R$.

Here, $R_{P}$ denotes the localization of $R$ at a prime ideal $P$.

Proof. To prove the first part of the lemma we recall the result about prime ideals mentioned in Section 2. Since a prime ideal which is not completely prime is paired with a c.prime ideal $P=P^{2} \neq(0)$ all prime ideals in a discrete right chain ring are c.prime. 
If $P$ is a c.prime ideal in a right chain ring and $S=R \backslash P$, then $N_{r}(S)=$ $\{a \in R \mid s a=0$ some $s \in S\}$ and $N_{l}(S)=\{a \in R \mid a s=0$ some $s \in S\}$ are ideals in $R$ and the set $\bar{S}$ consisting of the images of the elements of $S$ in $R / I=\bar{R}, I=N_{l}(S) \cup N_{r}(S)$ is a right Ore set in $\bar{R}$ and $\overline{R S}^{-1}=\left\{\overline{r s}^{-1} \mid \bar{r} \in\right.$ $\bar{R}, \bar{s} \in \bar{S}\}$ exists and is denoted by $R_{P}$.

If $Q$ is the maximal c.prime ideal in $R$ properly contained in $P$, then $I \subseteq Q=\bigcap_{n \in \mathrm{N}} P^{n}$ and $Q R_{P}$ is the maximal c.prime ideal in $R_{P}$ contained in $J\left(R_{P}\right)$ (as is common for commutative localizations, we write $Q$ for $\bar{Q}$ if there is no ambiguity).

Since $P R_{P}$ is two-sided, we have $\left(P R_{P}\right)^{2}=P^{2} R_{P} \neq P R_{P}$. To prove this last statement choose $m \in P \backslash P^{2}$ in $R$ and assume $\bar{m} \in P^{2} R_{P}$. This means $\bar{m}=\bar{p}_{1} \bar{p}_{2} \bar{s}^{-1}$ or $m s=p_{1} p_{2}+n$ in $R$ with $n \in I$ and $n=p_{1} n_{1}$ follows. Here, $n_{1} \in P$, since $\bar{n}=0 \neq \bar{p}_{1}$ shows that $n_{1} \in S$ is not possible. If $p_{1}=m t$ we obtain $m\left(s-t\left(p_{2}+n_{1}\right)\right)=0$ and $\bar{m}=0$, a contradiction. If $p_{1} t=m$, then $t \in S$ and $p_{1}\left(t s-\left(p_{2}+n_{1}\right)\right)=0$ and $\bar{p}_{1}=0=\bar{m}$, again a contradiction.

This shows that $\bar{m} \neq P^{2} R_{P}, P R_{P}=m R_{P}$ is a principal right ideal and $\bigcap\left(P R_{P}\right)^{i}=\cap m^{i} R_{P}=Q R_{P}$ and the only right ideals in $R_{P} / Q R_{P}$ are of the form $m^{i} R_{P} / Q R_{p},(0)$.

If $P$ is the minimal prime ideal of $R$, it could happen that $I=P$. Then $R_{P}$ is a division ring. Otherwise we show as before that $P R_{P} \neq P^{2} R_{P}$ and $R_{P}$ is right noetherian. (We choose $m \in P \backslash\left(P^{2} \cup I\right)$ for the argument as above.)

A partial converse of the last lemma is

LEMMA 3.10. Let $R$ be a right chain ring whose prime ideals are c.prime and satisfy the a.c.c. Further, assume that the ring $R_{P} / Q R_{P}$ is a right noetherian right chain ring for all pairs of neighbouring prime ideals $P \supset Q$. If $R$ is not a domain, assume $R_{P}$ also to be right noetherian for the minimal prime ideal $P$ of $R$. Then $R$ is discrete.

Proof. By Definition 3.8 it suffices to prove $P^{2} \neq P$ for all prime ideals $P \neq(0)$. We consider the situation $P \supset Q \neq(0)$, and denote by $m+Q_{P}$ the generator of the maximal (right) ideal in the right noetherian ring $R_{P} / Q R_{P}$. If we assume $P^{2}=P$, we obtain $m=m_{1} m_{2} \in P \backslash Q$ for some $m_{1}, m_{2} \in P$. On the other hand, $\left(m+Q_{P}\right)\left(r t^{-1}+Q_{P}\right)=m_{1}+Q_{P}$ holds for some $r t^{-1}+Q_{P}$, thus $m r t^{-1}-m_{1}=m_{1} m_{2} r t^{-1} \in Q_{P}$. The element $1-m_{2} r t^{-1}$ is a unit in the local ring $R_{P} / Q R_{P}$, thus $M_{1} \in Q_{P}$, a contradiction and $P^{2} \neq P$ follows. 
Now, consider the case where $P$ is the minimal completely prime ideal in $R$ and $R_{P}$ is right noetherian. If the kernel $I=N_{l}(S) \cup N_{r}(S)$ is strictly contained in $P$ we use similar arguments as above and we are done.

Therefore, we are left with the case $P=I+N_{l}(S) \cup N_{r}(S)$. First let $I=N_{l}(S)$ and $a \in P$, then $a s=0$ holds for some $s \in S$ which implies $a P=0$, thus $(0)=P^{2} \neq P$.

Now we consider the case $N_{r}(S)=I$ and assume $P^{2}=P$. If the segment (0) $\subset P$ is simple and if $X, Y$ are two-sided ideals with $X, Y \nsubseteq(0)$, we must have $P \subseteq X, Y$, and hence $(0) \subset P=P^{2} \subseteq X Y$. This shows that (0) is prime, by assumption even c.prime, and hence $R$ a domain. However, this leads to $N_{r}(S)=(0) \neq P$, a contradiction.

Therefore, it remains to discuss the case where $(0) \subset P$ is not simple and $P^{2}=P=N_{r}(S)$ is assumed. With the arguments as above we know that for each two-sided ideal $L$ with $(0) \subset L \subset P$ the segment $L \subset P$ is not simple. For every $a \in P$ we can find a two-sided ideal $L$ satisfying $a R \subseteq L \subset P$; to prove this, take the union $I$ of all two-sided ideals contained in $a R$. The union $I$ is again a two-sided ideal, thus, by the previous arguments there exists a two-sided ideal $L$ satisfying $a R \subseteq L \subset P$. The intersection $\cap L^{n}$ of a two-sided ideal which is not nilpotent is c.prime, therefore we conclude $L^{n}=(0)$ for some $n \in \mathbb{N}$ as $P$ is the minimal prime ideal and finally $a^{n}=0$ holds for some $n \in \mathbb{N}, a$ arbitrarily chosen in $P$. Now we want to show that even $P^{3}=0$ which would lead to $P=(0)$, a contradiction. Take $x, y, z \in$ $P$. As $I=N_{r}(S)$ holds we have $s x=0$ for some $s \in S$. Let $s r=x$ for some $r \in P$ and $x y z=s r y z$ follows. If $r y=y r_{1}$ holds for a suitable $r_{1} \in R$, we are done. Otherwise $r y r_{1}=y$ follows for some $r_{1} \in R$. If $r_{1} R \supseteq z R$ then $x y z=s r y z=s r y r_{1} r_{2}=s y r_{2}=0$ with $r_{1} r_{2}=z$. However, $r_{1}=z r_{2}$ leads to $r y z r_{2}=y$ and thus $r^{n} y\left(z r_{2}\right)^{n}=y$, a contradiction as $z r_{2} \in P$ implies $\left(z r_{2}\right)^{n}=0$ for a sufficiently large $n$.

Discrete right chain rings need not to be right invariant as the following example shows.

If one denotes by $V_{1}$ and $V_{2}$ the two extensions of $\mathbb{Z}_{(5)}$, the localization of $\mathbb{Z}$ at $(5)$ in $\mathbb{Z}[i]$ and with $\sigma$ conjugation in $\mathbb{Q}(i)$, then

$$
R=\left\{\sum_{k=0}^{\infty} t^{k} a_{k} \mid a_{k} \in \mathbb{Q}(i), a_{0} \in V_{1}\right\} \subseteq \mathbb{Q}(i)[[t, \sigma]]
$$

is a non right invariant discrete valuation ring; here $\mathbb{Q}(i)[[t, \sigma]]$ is the skew power series ring in one variable $t$ with coefficients in $\mathbb{Q}(i)$ and at $=t a^{\sigma}$ defines the multiplication. If we assume that $V_{1}=\mathbb{Z}[i]_{(2-i)}$ then $\frac{1}{2+i} t \notin t R$ 
since $t^{-1} \frac{1}{2+i} t=\frac{1}{2-i} \notin R$. However, the two prime ideals $P_{1}=(2-i) R=$ $J(R)$ and $P_{2}$ generated by $\left\{t(2-i)^{-n} \mid n=1,2, \ldots\right\}$ are complete and satisfy $P_{i}^{2} \neq P_{i}-R$ is a discrete right chain ring that is not right invariant.

LEMMA 3.11. If $R_{1}$ is an immediate extension of a discrete right chain ring $R$ then $R_{1}$ is discrete.

Proof. $R_{1}$ cannot contain a prime ideal $Q$ that is not completely prime, since in that case a c.prime ideal $P$ would exist in $R_{1}$ and $P^{2}=P$. The intersection $P \cap R$ would be an idempotent c.prime ideal in $R$ (Proposition 3.4(ix)) - a contradiction to the assumption. Applying this last argument again, we conclude that all complete prime ideals $\neq(0), R$ in $R_{1}$ are not idempotent. If $P_{1}, P_{2}$ are distinct c.prime ideals in $R_{1}$ then $R \cap P_{2}, R \cap P_{2}$ are distinct c.prime ideals in $R$ and hence a.c. for c.prime ideals follows for $R_{1}$.

We conclude this section with an open problem.

Open problem. If $R_{1}$ is an immediate extension of the right chain ring $R$ and $I$ a two-sided ideal of $R$ is then $I R_{1}$ a two-sided ideal of $R_{1}$ ?

\section{Pseudo convergent sequences and linear compactness}

The elements in an immediate extension $R_{1}$ of a right chain ring $R$ that are not contained in $R$ can be described as limits of pseudo convergent sequences in $R$. If every such sequence in $R$ has already a limit in $R$, then $R$ is called maximally complete. Some of the definitions extend to uniserial modules.

Let $M_{R}$ be a uniserial $R$-module, $R$ a ring. We define a mapping $v$ from $M_{R} \backslash\{0\} \mid$ onto the set $\mathscr{W}_{M}=\{a R \mid 0 \neq a \in M\}$ by $v(a)=a R$ and set $v(a) \geq$ $v(b)$ if and only if $a R \subseteq b R$. It follows that $v(a-b) \geq \min \{v(a), v(b)\}$ with equality if $v(a) \neq v(b)$.

The definitions of pseudo convergent sequences and limits as considered by Ostrowski, Kaplansky, Schilling and others extend, as do the basic propositions, to uniserial modules.

Definition 4.1. Let $M_{R}$ be a uniserial $R$-module. A sequence $\left(a_{\rho}\right)_{\rho \in \Lambda}$, $a_{\rho} \in M_{R}$ and $\Lambda$ well-ordered with no last element is called pseudo convergent (p.c.) if $v\left(a_{\tau}-a_{\sigma}\right)>v\left(a_{\sigma}-a_{\rho}\right)$ for $\rho<\sigma<\tau \in \Lambda$.

LEMMA 4.2. If $\left(a_{\rho}\right)_{\rho \in \Lambda}$ is a p.c. sequence in $M_{R}$, then either

(i) $v\left(a_{\rho}\right)<v\left(a_{\sigma}\right)$ for $\rho<\sigma$ or

(ii) $v\left(a_{\rho}\right)=v\left(a_{\sigma}\right)$ for $\rho, \sigma \geq \lambda$ and some ordinal $\lambda$. 
Proof. Suppose (i) does not hold, that is $v\left(a_{\rho}\right) \geq v\left(a_{\sigma}\right)$ for some $\rho<\sigma$. Then $v\left(a_{\tau}\right)=v\left(a_{\sigma}\right)$ for all $\tau>\sigma$, since otherwise

$$
v\left(a_{\tau}-a_{\sigma}\right)=\min \left\{v\left(a_{\tau}\right), v\left(a_{\sigma}\right)\right\} \leq v\left(a_{\sigma}\right),
$$

but $v\left(a_{\sigma}-a_{\rho}\right) \geq v\left(a_{\sigma}\right)$-a contradiction

LEMMA 4.3. Let $\left(a_{\rho}\right)_{\rho \in \Lambda}$, be a p.c. sequence in $M_{R}$. Then $v\left(a_{\sigma}-a_{\rho}\right)=$ $v\left(a_{\rho+1}-a_{\rho}\right)$ for all $\sigma>\rho$.

Proof. We can assume that $\sigma>\rho+1$ and obtain

$$
v\left(a_{\sigma}-a_{\rho+1}\right)>v\left(a_{\rho+1}-a_{\rho}\right)
$$

and $a_{\sigma}-a_{\rho}=\left(a_{\sigma}-a_{\rho+1}\right)+\left(a_{\rho+1}-a_{\rho}\right)$. It follows that

$$
v\left(a_{\sigma}-a_{\rho}\right)=\min \left\{v\left(a_{\sigma}-a_{\rho+1}\right), v\left(a_{\rho+1}-a_{\rho}\right)\right\}=v\left(a_{\rho+1}-a_{\rho}\right) .
$$

Let $\left(a_{\rho}\right)_{\rho \in \Lambda}$ be a p.c. sequence. We write $v\left(a_{\sigma}-a_{\rho}\right)=\gamma_{\rho}$ where $\gamma_{\rho} \in \mathscr{W}_{M}$ is a constant for all $\sigma>\rho$ by the above lemma.

Definition 4.4. An element $x \in M_{R}$ is called a limit of the p.c. sequences $\left(a_{\rho}\right)_{\rho \in \Lambda}$, if $v\left(x-a_{\rho}\right)=\gamma_{\rho}$ for all $\rho$.

Definition 4.5. The submodule $B$ of $M$ consisting of elements $b \in M_{R}$ with $v(b)>\gamma_{\rho}$ for all $\rho$ is called the breadth of the p.c. sequence $\left(a_{\rho}\right)_{\rho \in \Lambda}$.

Let $\left(a_{\rho}\right)_{\rho \in \Lambda}$ be a p.c. sequence of type (i) as described in Lemma 4.2. Then an element $b \in M$ is a limit of $\left(a_{\rho}\right)$ if and only $b$ is in the breadth of $\left(a_{\rho}\right)$ : we observe that $v\left(a_{\sigma}-a_{\rho}\right)=v\left(a_{\rho}\right)=\gamma_{\rho}$ for $\rho<\sigma$ then $v\left(b-a_{\rho}\right)=\gamma_{\rho}$ and $b$ is a limit. Conversely, if $v\left(x-a_{\rho}\right)=\gamma_{\rho}=v\left(a_{\rho}\right)$ then $v(x) \geq \gamma_{\rho}$ for all $\rho$ and hence $v(x) \geq \gamma_{\rho+1}>\gamma_{\rho}$, that is, $x$ is in the breadth of $\left(a_{\rho}\right)$.

With similar arguments one proves the following result.

Lemma 4.6. Let a be a limit of a p.c. sequence $\left(a_{\rho}\right)$ in $M_{R}$. Then $c \in M_{R}$ is a limit of $\left(a_{p}\right)$ in $M$ if and only if $c=a+b$ for $b \in B$, the breadth of $\left(a_{\rho}\right)$.

Definition 4.7. We say a uniserial right $R$-module $M$ is maximally complete if every p.c. sequence in $M$ has a limit in $M$.

Let $M_{R}$ be an uniserial $R$-module, $I_{\alpha} \subseteq M_{R}, \alpha \in \Lambda$, a family of submodules of $M$ where $\Lambda$ is a well-ordered set of indices and $I_{\alpha} \supset I_{\beta}$ for $\alpha<\beta$. Further, let $\left(x_{\alpha}\right)_{\alpha \in \Lambda}$ be à sequence of elements in $M_{R}$ and $\mathscr{T}=\left(x_{\alpha}+I_{\alpha}\right)_{\alpha \in \Lambda}$ the corresponding sequence of cosets in $M$. 
DEFINITION 4.8. With $M_{R}, I_{\alpha}, \Lambda,\left(x_{\alpha}\right)$ as above we have the following definitions.

(i) $\mathscr{T}$ has the finite intersection property $(F I P)$ if $\bigcap_{\alpha \in \mathscr{F}}\left(x_{\alpha}+I_{\alpha}\right) \neq \varnothing$ for all finite subsets $\mathscr{F} \subseteq \Lambda$.

(ii) $\mathscr{T}$ is pairwise solvable if $\left(x_{\alpha}+I_{\alpha}\right) \cap\left(x_{\beta}+I_{\beta}\right) \neq \varnothing$ for all $\alpha, \beta \in \Lambda$; we also say that $\mathscr{T}$ is compatible.

(iii) An element $a \in \bigcap_{\alpha \in \Lambda}\left(x_{\alpha}+I_{\alpha}\right)$ is called a solution of $x \equiv x_{\alpha} \bmod I_{\alpha}$ for all $\alpha \in \Lambda$; we also say that $a$ is a solution for $\mathscr{T}$.

(iv) The intersection $\cap I_{\alpha}=B$ is called the breadth of $\mathscr{T}$.

(v) Let $B$ be a submodule of $M_{R}$. Then $M_{R}$ is called linearly $B$-compact if all sequences $\mathscr{T}$ with breadth $B$ have a solution.

(vi) $M$ is called (almost) linearly compact if $M$ is linearly $B$ compact for all submodules $B \subseteq M(B \neq(0))$.

(vii) A ring is called (almost) right linearly compact if $R_{R}$ is (almost) right linearly compact.

Remark. If $B$ is a submodule of the uniserial module $M_{R}$ which has an immediate upper neighbour $B^{\prime} \supset B$ in the lattice of submodules of $M$ then any sequence $\mathscr{T}=\left(x_{\alpha}+I_{\alpha}\right)_{\alpha \in \Lambda}$, with breadth $\mathscr{T}=\bigcap I_{\alpha}=B$, satisfies $I_{\alpha}=B$ for some $\alpha$, that is, $\Lambda$ has a last element.

LeMma 4.9. Let $M_{R}, \Lambda,\left(x_{\alpha}\right),\left(I_{\alpha}\right)_{\alpha \in \Lambda}$, and $\mathscr{T}$ be as above. Then the following conditions are satisfied.

(i) The sequence $\mathscr{T}$ is pairwise solvable if and only if $x_{\alpha}-x_{\beta} \in I_{\alpha} \cup I_{\beta}$ for all $\alpha, \beta \in \Lambda$.

(ii) $\mathscr{T}$ is pairwise solvable if and only if $\mathscr{T}$ has FIP.

(iii) The sequence of cosets $\left(x_{\alpha}+I_{\alpha}\right)_{\alpha \in \Lambda}$ is linearly ordered by inclusion if $\mathscr{T}$ is pairwise solvable.

(iv) If all compatible sequences $\mathscr{T}$ with breadth $I$ and cyclic $R$-modules $I_{\alpha}$ are solvable, then all compatible sequences $\mathscr{T}$ with breadth $I$ are solvable.

(v) If $x, x^{\prime} \in M_{R}$ are solutions for $\mathscr{T}$, then $x-x^{\prime} \in I$ where $I$ is the breadth of $\mathscr{T}$.

Proof. (i) If $T$ is pairwise solvable there exists an element $x \in M$ with $x-x_{\alpha} \in I_{\alpha}, x-x_{\beta} \in I_{\beta}$ and $x_{\alpha}-x_{\beta} \in I_{\alpha} \cup I_{\beta}=I_{\alpha}$ for $\alpha \leq \beta$. On the other hand, if $x_{\alpha}-x_{\beta} \in I_{\alpha} \supseteq I_{\beta}$ then $x_{\beta}-x_{\alpha} \in I_{\alpha}$ and $x_{\beta} \in\left(x_{\beta}+I_{\beta}\right) \cap\left(x_{\alpha}+I_{\alpha}\right)$.

(ii) Let $I_{\alpha_{1}} \supset I_{\alpha_{2}} \supset \cdots \supset I_{\alpha_{n}}$ for $\alpha_{1}<\alpha_{2}<\cdots<\alpha_{n}$. Then $x_{\alpha_{n}}-x_{\alpha_{i}} \in I_{\alpha_{i}}$ for $i=1, \ldots, n$, if we assume pairwise solvability.

(iii) Suppose $x_{\alpha}+I_{\alpha}, x_{\beta}+I_{\beta}$ are given with $I_{\alpha} \supset I_{\beta}$ and $x_{\beta}-x_{\alpha} \in I_{\alpha}$. Then $x_{\beta}+I_{\beta} \supseteq x_{\alpha}+I_{\alpha}$ follows immediately 
(iv) Let $\mathscr{T}=\left(x_{\alpha}+I_{\alpha}\right)_{\alpha \in \Lambda}$, be a compatible sequence. The statement is trivial if $\Lambda$ has a last element. Otherwise consider $x_{\alpha}-x_{\alpha+1}=a_{\alpha}^{\prime} \in I_{\alpha}$ for every $\alpha \in \Lambda$, and choose $a_{\alpha} \in M$ with $I_{\alpha+1} \cup a_{\alpha}^{\prime} R \supseteq a_{\alpha} R \supseteq I_{\alpha}$ and $y_{\alpha}=x_{\alpha+1}$. Then $\bigcap a_{\alpha} R=\bigcap I_{\alpha}$ and $\bigcap\left(y_{\alpha}+a_{\alpha} R\right)=\bigcap\left(x_{\alpha}+I_{\alpha}\right)$ where $\mathscr{T}^{\prime}=\left(y_{\alpha}+a_{\alpha} R\right)$ is a compatible sequence.

LEMMA 4.10. Let $M_{R}$ be a uniserial R-module which is linearly I-compact. Then the $R$-module $M / N$ is linearly $I / N$-compact for every submodule $N$ of $M$ with $N \subseteq I$.

Proof. Let $\left(x_{\alpha}+N_{\alpha} / N\right)_{\alpha \in \Lambda}$ be a compatible sequence. Then, obviously $\left(x_{\alpha}+N_{\alpha}\right)_{\alpha \in \Lambda}$ is compatible as $N \subseteq N_{\alpha}$ holds for all $\alpha$. The image of a solution $\left(x_{\alpha}+N_{\alpha}\right)_{\alpha \in \Lambda}$ yields a solution of the given sequence.

We state the next theorem without proof since the original proof for commutative valuation domains can be directly adapted to our situation (see [10]).

THEOREM 4.11. Let $M_{R}$ be a uniserial module and $\left(a_{\rho}\right)_{\rho \in \Lambda}$ a p.c. sequence in $M_{R}$ with breadth $B$. Then there exists a compatible sequence $\mathscr{T}$ of cosets in $M_{R}$ with the same $B$ and the set of limits of $\left(a_{\rho}\right)$ is equal to the set of solutions for $\mathscr{T}$. Conversely, for any compatible sequence $\mathscr{T}=\left(x_{p}+I_{p}\right)_{p \in \Lambda}$ with breadth $B$ there exists. a subset $\Lambda_{0} \subseteq \Lambda$ such that $\left(x_{\rho}\right)_{\rho \in \Lambda_{0}}$ is a p.c. sequence with breadth $B$ and $\bigcap_{p \in \Lambda}\left(x_{p}+I_{\rho}\right)$ is the set of limits of $\left(x_{\rho}\right)_{p \in \Lambda_{0}}$ or $x_{\tau} \in \bigcap\left(x_{\rho}+I_{\rho}\right)$ for some $\tau \in \Lambda$.

This results shows that a uniserial module $M$ is maximally complete if and only if it is linearly compact or more specifically, every p.c. sequence in $M$ with breadth $I$ has a limit in $M$ if and only if $M$ is linearly $I$-compact.

The next result shows that maximally complete right chain rings are maximal; it generalizes a theorem by Kaplansky; see [5].

THEOREM 4.12. Let $R$ be a right chain ring and $R_{1}$ an immediate extension of $R$. Then every element $z \in R_{1} \backslash R$ is a limit of a p.c. sequence in $R$ that has no limit in $R$.

Proof. Consider the subset $S=\{v(z-a) \mid a \in R\} \subseteq \mathscr{W}$. This is welldefined since $z-a \neq 0$ for all $a \in R$. Further, $S$ cannot have a largest element, $w$ say. Otherwise $v(z-a)=w$ and $z-a=b u$, for $b \in R$, $u \in U\left(R_{1}\right)$ and some $a \in R$. However $u=d+j_{1}$ where $d$ is a representative of $u+J\left(R_{1}\right)$ in $R$ and $j_{1} \in J\left(R_{1}\right)$. It follows that $z-a-b d=b j_{1}$ and $v(z-a-b d)>v(z-a)$. 
We can therefore select a well-ordered cofinal sequence in $S$ and the corresponding sequence $\left(a_{\rho}\right)_{p \in \Lambda}$ and $a_{\rho} \in R$ and $\Lambda$ well-ordered. We have $v\left(z-a_{\sigma}\right)<v\left(z-a_{\rho}\right)$ by definition, $v\left(\alpha_{\sigma}-a_{\rho}\right)=v(z-a \rho)$ follows and no last element exists in $\left\{z-a_{\rho} \mid \rho \in \Lambda\right\}$ and $\left(a_{\rho}\right)_{\rho \in \Lambda}$ is a p.c. sequence in $R$ with $z$ one of its limits in $R_{1}$. If we assume that $z_{1}$ is a limit of $\left(a_{\rho}\right)_{\rho \in \Lambda}$ in $R$ then $v\left(z-z_{1}\right)=v\left(\left(z-a_{\rho}\right)-\left(z_{1}-a_{\rho}\right)\right) \geq \min \left\{v\left(z-a_{\rho}\right), v\left(z_{1}-a_{\rho}\right)\right\}=\gamma_{\rho}=v\left(z-a_{\rho}\right)$ for all $\rho$. This contradicts the fact that $\left\{v\left(z-a_{\rho}\right) \mid \rho \in \Lambda\right\}$ is cofinal in $S$.

We have the following partial converse of Theorem 4.12.

THEOREM 4.13. Let $R \subseteq R_{1}$ be right chain rings with $J(R)=J\left(R_{1}\right) \cap R$ such that for every $x \in R_{1} \backslash R$ is a limit of a p.c. sequence in $R$ which has no limit in $R$. Then $R_{1}$ is an immediate extension of $R$.

Proof. We observe that $a R \neq b R, a, b \in R$, implies $a R_{1} \neq b R_{1}$ since $J(R)-J\left(R_{1}\right) \cap R$. Otherwise $a u=b, u \in U\left(R_{1}\right)$ and $a j=b$, $j \in J(R) \subseteq J\left(R_{1}\right)$ and $a(u-j)=0, u-j \in U\left(R_{1}\right), a=0$. A p.c. sequence $\left(a_{\rho}\right)$ in $R$ is also a p.c. sequence in $R_{1}$. We use Lemma 3.3 and it is enough to show that for $x \in R_{1} \backslash R$ we have $x R_{1}=a R_{1}$ for some $a \in R$, since then $x=a u_{1}=a\left(u+j_{1}\right)=a u\left(1+u^{-1} j_{1}\right)$ for $u_{1} \in U\left(R_{1}\right)$ and $u-u_{1} \in J\left(R_{1}\right)$ for some $u \in R \cap U\left(R_{1}\right)=U(R)$, since $u_{1}$ is in $R$ or the limit of a p.c. sequence in $R$. The element $x$ is the limit in $R_{1}$ of a c.c. sequence $\left(a_{\rho}\right)$ of $R$ which has no limit in $R$. Hence, $v\left(a_{\rho}\right)=v\left(a_{\sigma}\right)$ for any $\sigma>\rho \geq \lambda$ for some index $\lambda$ using Lemma 4.2 and the remark after Definition 4.5. If $x=a_{\rho} j_{1}$ for $j_{1} \in J\left(R_{1}\right)$ then also $x=a_{\sigma} j_{2}$ for some $j_{2} \in J\left(R_{1}\right)$ and $v\left(x-a_{\rho}\right)=v\left(a_{\rho}\right)=v\left(a_{\sigma}\right)=v\left(x-a_{\sigma}\right)$, a contradiction. Conversely, if $x j_{1}=a_{\rho}, j_{1} \in J\left(R_{1}\right)$, then also $x j_{2}=a_{\sigma}$ for some $j_{2} \in J\left(R_{1}\right)$ and $v\left(x-a_{\rho}\right)=v(x)=v\left(x-a_{\sigma}\right)$, again a contradiction. This leaves $x R_{1}=a_{\sigma} R_{1}$ as the only possibility and proves the lemma.

Proposition 4.14. Let $R \subset R_{1}$ be a proper immediate extension and $x \in R \backslash R_{1}$ a limit of a p.c. sequence $\left(a_{p}\right)_{p \in \Lambda}$ in $R$ with breadth $B$ with no limit in $R$. Then $R / I \subset R_{1} / I R_{1}$ for every right ideal $I \subseteq B$.

Proof. The p.c. sequence $\left(a_{\rho}\right)$ in $R$ is a p.c. sequence in $R_{1}$ with breadth $B R_{1}$ if $B$ is its breadth in $R$. If $z$ is an element in $R$ with $z+B R_{1}=$ $x+B R_{1}$ then $z=x+b_{1}, b_{1} \in B R_{1}$, is a limit of $\left(a_{p}\right)$ in $R$ (Lemma 4.6), a contradiction; $R / I \subset R_{1} / I R_{1}$ for every right ideal $I \subset B$ follows.

We conclude this section with a result that provides some information on the set of right ideals $I^{\prime}$ of a right chain ring $R$ for which $R$ is linearly 
$I^{\prime}$-compact provided $R$ is linearly $I$-compact. Right ideals $A, B$ of $R$ are called related if $s^{-1} A=t^{-1} B$ for some elements $s, t \in R$ with $s \notin A$, $t \notin B$, where $s^{-1} A=\{x \in R \mid s x \in A\}$. It is proved that $R$ is linearly $B$-compact provided $R$ is $A$-compact and $B$ is related to $A$. However the general problem seems to be open.

Open problem. Assume that $R$ is $A$-compact for all $A \in \mathscr{A}$ with $\mathscr{A}$ a subset of the set of right ideals of $R$. Describe all right ideals $B$ for which $R$ must be $B$-compact too.

THEOREM 4.15. Let $R$ be a right chain ring and $A, B$ right ideals satisfying $s^{-1} A=t^{-1} B$ for some $s \notin A, t \notin B$. Then the following conditions are equivalent:

(i) $R$ is linearly $A$-compact.

(ii) $R$ is linearly $B$-compact.

Proof. It is sufficient to prove that $A$-compactness is equivalent to $s^{-1} A$-compactness, $s \notin A$ and we assume first that $R$ is linearly $A$-compact and $\mathscr{T}=\left(x_{\alpha}+X_{\alpha}\right)_{\alpha \in \Lambda}$ a compatible sequence of cosets with $\bigcap X_{\alpha}=s^{-1} A$. Then $\left(s x_{\alpha}+s X_{\alpha}\right)_{\alpha \in \Lambda}$ is a compatible sequence of cosets with breadth $A$ and hence a solution $a$ exists.

If $a=s x$ for some $x \in R$, then $s\left(x-x_{\alpha}\right) \in s X_{\alpha}, x-x_{\alpha}-n \in X_{\alpha}$ for $n \in R$ with $s n=0$. Hence, $n \in s^{-1} A \subseteq X_{\alpha}$ and $x$ is a solution for $\mathscr{T}$.

If $a q=s$ for some $q \in J(R)$, then $a-a q x_{\alpha}=a\left(1-q x_{\alpha}\right) \in s X_{\alpha}$ and $a \in s X_{\alpha}$ follows for all $\alpha \in \Lambda$. We have $a \in s X_{\alpha}, s \in s X_{\alpha}$ and $s \in \bigcap s X_{\alpha}=A$, a contradiction.

If conversely every compatible system with breadth $s^{-1} A$ is solvable let $\mathscr{T}_{1}=\left(a_{\alpha}+A_{\alpha}\right)_{\alpha \in \Lambda}$ be a compatible sequence with $\bigcap A_{\alpha}=A$. There exists an index $\alpha_{0}$ with $A_{\alpha_{0}} \subset s R$ and we define $\Lambda^{\prime}=\left\{\alpha \mid \alpha \geq \alpha_{0}\right\}$. It is sufficient to show that $\left(a_{\alpha}+A_{\alpha}\right)_{\alpha \in \Lambda^{\prime}}$ has a solution. Since $a_{\alpha}-a_{\alpha_{0}} \in A_{\alpha_{0}}, \alpha \in V^{\prime}$, there exists $t_{\alpha} \in R$ with $a_{\alpha}-a_{\alpha_{0}}=s t_{\alpha}$. The system $\mathscr{T}=\left(t_{\alpha}+s^{-1} A_{\alpha}\right)_{\alpha \in \Lambda^{\prime}}$ has $\cap s^{-1} A_{\alpha}=s^{-1} \cap_{\alpha \in \Lambda^{\prime}} A_{\alpha}$ as its breadth and is compatible, since $s\left(t_{\alpha}-t_{\beta}\right)=$ $\left(a_{\alpha}-a_{\alpha_{0}}\right) \in A_{\alpha}$ for $\alpha \leq \beta$. Hence, a solution $t$ exists for $\mathscr{T}$ and $a_{\alpha_{0}}+s t$ is a solution for $\mathscr{T}_{1}$.

\section{A counterexample}

We saw that every element $x \in R_{1} / R$, with $R_{1}$ maximal immediate extension of a right chain ring $R$, is the limit of a p.c. sequence in $R$. We 
show now by an example that a right chain ring $R$ can be maximal without being maximally complete.

Consider $R=\mathbb{Q}\left(t_{1}, t_{2}, \ldots\right)[x]_{(x)}[[y, \sigma]]$ with $x y=y t_{1}, t_{i} y=y t_{i+1}$ where $\sum_{n=0}^{\infty} y^{n} f_{n}\left(t_{i}, x\right)$ are the elements of $R, \mathbb{Q}\left(t_{1}, t_{2}, \ldots\right)$ is a function field in the variables $t_{i}, i=1, \ldots$, over the rational numbers $\mathbb{Q}$, and $\mathbb{Q}\left(t_{1}, t_{2}, \ldots\right)[x]_{(x)}$ is the localization at $(x)$ of the polynomial ring in one variable $x$ over $\mathbb{Q}\left(t_{1}, t_{2}, \ldots\right)$ containing the coefficients $f_{n}\left(t_{i}, x\right)$. The ring $R$ is then a skew power series ring over this ring in the variable $y$ with $\sigma(x)=t_{1}, \sigma\left(t_{i}\right)=t_{i+1}$ and is a right invariant right chain domain with its right ideals $\neq(0)$ of the form $y^{n} x^{m} R, n, m \geq 0$.

We show that there cannot exist a proper immediate extension $R_{1}$ of $R$. Otherwise, $R_{1}$ is again right noetherian, and hence right invariant with right ideals $y^{n} x^{m} R_{1}$ (by Proposition 3.7(v), Lemma 3.4(iv)). By Theorem 4.12, $R_{1}$ contains an element $f, f \notin R$, which is a pseudo limit of a p.c. sequence $\left(a_{\rho}\right)_{\rho \in \Lambda}$ of elements $a_{\rho}$ in $R$ with breadth $B$ and which has no limit in $R$. Since $B$ is a right ideal without an upper neighbour in the lattice of right ideals, it follows that $B$ is either $(0)$ or $y^{i} R, i=1, \ldots$, However, $R$ is linearly (0)-compact by construction and if $R$ is not $y^{n} R$-compact for some $n$, then $R$ is not $y R$-compact, from Theorem 4.15. Finally, using Proposition 4.14 , we can assume that $R / y R$, which is a ring, is contained in but is not equal to $R_{1} / y R_{1}$, where we use $y R_{1} \cap R=y R$ by Proposition $3.4(\mathrm{vi})$, that is, we can assume that the image of the element $f$ in $R_{1} / y R_{1}$ is not contained in $R / y R$.

Next, let $g$ be any element in $R_{1} \backslash y R_{1}$. Then $g$ can be written as $g=$ $x^{k}\left(c_{0}+x h\right)$ where $k \geq 0$ is an integer, $c_{0} \in \mathbb{Q}\left(t_{1}, t_{2}, \ldots\right) \simeq R / J(R) \simeq$ $R_{1} / J\left(R_{1}\right), c_{0} \neq 0, h \in R_{1}$ and $c_{0}+x h=u$ is a unit in $R_{1}$. We consider $g y=x^{k}\left(c_{0}+x h\right) y=x^{k} u y-y t_{1}^{k} u^{\prime}=y g^{\prime}$ with $u^{\prime} \in U\left(R_{1}\right), g^{\prime} \in R_{1}$ and $g^{\prime}=d_{0}+x h_{1} \in U\left(R_{1}\right)$ follows for some $h_{1} \in R_{1}$ with $0 \neq d_{0} \in \mathbb{Q}\left(t_{1}, \ldots\right)$. The element $d_{0}$ is uniquely determined by $g$ and we define a mapping $\Psi$ from $R_{1}$ to $\mathbb{Q}\left(t_{1}, \ldots\right)$ by $\Psi(g)=d_{0}$ as defined above if $g \in R_{1} \backslash y R_{1}$ and $\Psi(g)=0$ otherwise. It follows that $\Psi\left(g_{1}\right)+\Psi\left(g_{2}\right)=\Psi\left(g_{1}+g_{2}\right)$ for $g_{1}$, $g_{2} \in R_{1}$ and we prove $\Psi\left(g_{1}, g_{2}\right)=\Psi\left(g_{1}\right) \Psi\left(g_{2}\right)$. Let $g_{1} y=y\left(d_{0}+x h_{1}\right)$, $g_{2} y=y\left(d_{0}^{\prime}+x h_{2}\right)$ for $d_{0}, d_{0}^{\prime} \in \mathbb{Q}\left(t_{1}, \ldots\right), h_{1}, h_{2} \in R_{1}$, then $g_{2} g_{1} y=$ $y\left(d_{0}^{\prime}+x h_{2}\right)\left(d_{0}+x h_{1}\right)=y\left(d_{0}^{\prime} d_{0}+x w\right)$ for a certain element $w \in R_{1}$, that is, $\Psi\left(g_{2} g_{1}\right)=d_{0}^{\prime} d_{0}=\Psi\left(g_{2}\right) \Psi\left(g_{1}\right)$ and $\Psi$ is a ring homomorphism from $R_{1}$ to $\mathbb{Q}\left(t_{1}, \ldots\right)$ with kernel $y R_{1}$.

Consider $f \in R_{1} \backslash R, f \notin y R_{1}$ as chosen above. Then

$$
\Psi(f) \in \mathbb{Q}\left(t_{1}, \ldots\right) \backslash \mathbb{Q}\left(t_{2}, \ldots\right)\left[t_{1}\right]_{\left(t_{1}\right)}
$$

since $\Psi\left(\mathbb{Q}\left(t_{1}, \ldots\right)[x]_{(x)}\right)=\mathbb{Q}\left(t_{2}, \ldots\right)\left[t_{1}\right]_{\left(t_{1}\right)}$ and $R / y R=\mathbb{Q}\left(t_{1}, \ldots\right)[x]_{(x)} \subset$ 
$R_{1} / y_{1} R$. This implies $\Psi(f)=t_{1}^{-k} h_{1}\left(t_{i}\right) / g_{1}\left(t_{i}\right)$ with $k \geq 1, h_{1}\left(t_{i}\right), g_{1}\left(t_{i}\right) \in$ $\mathbb{Q}\left[t_{1}, \ldots\right]$ and $\left(t_{1}, h_{1}\left(t_{i}\right) g_{1}\left(t_{i}\right)\right)=1$. There exists $g$ in $R$ with

$$
\Psi(g)=g_{1}\left(t_{i}\right) / h_{1}\left(t_{i}\right) \in \mathbb{Q}\left(t_{2}, \ldots\right)\left[t_{1}\right]_{\left(t_{1}\right)}
$$

and it follows that

$$
\Psi\left(x^{k} g f-1\right)=\Psi\left(x^{k}\right) \Psi(g) \Psi(f)-1=t_{1}^{k} \cdot \frac{g_{1}\left(t_{i}\right)}{h_{1}\left(t_{i}\right)} \cdot t_{1}^{-k} \frac{h_{1}\left(t_{i}\right)}{g_{1}\left(t_{i}\right)}-1=0
$$

and $x^{k} g f=1+y r^{\prime}$ for some $r^{\prime} \in R_{1}$. This shows that $x^{k}$ is a unit for some $k>0$, a contradiction.

\section{References}

[1] C. Bessenrodt, H. H. Brungs and G. Törner, 'Prime ideals in right chain rings,' Mitt. Math. Sem. Giessen 163 (1984), 141-167.

[2] C. Bessenrodt, H. H. Brungs and G. Törner, 'Right chain rings.' Part 1, FB Mathematik, Preprint No. 74, Universität Duisburg, 1985.

[3] W. Brandal, 'Almost maximal integral domains and finitely generated modules,' Trans. Amer. Math. Soc. 183 (1973), 203-222.

[4] N. I. Dubrovin, 'An example of a nearly simple chain ring with nilpotent elements,' (Russian) Mat. Sb. (N.S.) 120 (1983), 441-447.

[5] I. Kaplansky, 'Maximal fields with valuations,' Duke Math. J. 9 (1942), 303-321.

[6] I. Kaplansky, 'Modules over. Dedekind rings and valuation rings,' Trans. Amer. Math. Soc. 72 (1952), 327-340.

[7] W. Krull, 'Allgemeine Bewertungstheorie,' J. Reine Angew. Math. 167 (1932), 160-196.

[8] K. Mathiak, Valuations of skew fields and projective Hjelmslev spaces, (Lecture Notes in Mathematics 1175, Springer, Berlin, 1986).

[9] F. J. Rayner, 'Gravett's proof of a theorem of Krull', Quart. J. Math. 24 (1973), 409410.

[10] O. Schilling, The theory of valuation, (Math. Surveys, Amer. Math. Soc., Providence, R. I., 1950).

University of Alberta

Edmonton, Alberta

Canada T6G 2G1
Fachbereich Mathematik

Universität Duisburg

D 4100 Duisburg

Federal Republic of Germany 